\title{
PREVALÊNCIA DA SÍNDROME DE BURNOUT E QUALIDADE DO SONO EM TRABALHADORES TÉCNICOS DE ENFERMAGEM
}

\section{Julio Simões}

Enfermeiro; Especialista em Anatomia e Histologia pela Universidade Estadual de Maringá (UEM), Maringá (PR), Brasil.

E-mail: juliosimoes2000@yahoo.com.br

\section{Larissa Renata de Oliveira Bianchi}

Docente Doutora do Departamento Ciências Morfologia da Universidade Estadual de Maringá (UEM), Maringá (PR), Brasil.
RESUMO: A Síndrome de Burnout e a má qualidade do sono têm se propagado com mais facilidade entre técnicos de enfermagem. $\mathrm{O}$ objetivo deste estudo foi avaliar a prevalência da Síndrome de Burnout e a qualidade do sono em técnicos de enfermagem. Essa pesquisa foi realizada com 47 técnicos de enfermagem, com trabalham no plantão noturno. Foram aplicados os questionários: 1 socioeconômico, $2 \mathrm{MBI}$ Maslach Burnout Inventory e 3 PSQI - Pittsburgh Sleep Quality Index. Com base nos inventários utilizados, $16,33 \%$ apresentaram possíveis sinais e sintomas de Burnout, $61,73 \%$ alto índice para manifestação de Burnout, enquanto 21,93\% apresentaram baixo índice para esta patologia. Em relação à Qualidade de Sono no último mês, $74,4 \%$ apresentaram uma má qualidade de sono e $25,5 \%$ uma boa qualidade de sono. A ocorrência de Burnout e a má Qualidade de Sono na população pesquisada foi elevada, possivelmente em função dos horários em turno noturno, bem como jornadas de até três turnos de trabalho em busca de salários mais satisfatórios. Conscientizar a importância do sono e de hábitos que reduzam o estresse é a saída para amenizar os efeitos colaterais na vida pessoal e profissional.

PALAVRAS-CHAVE: Esgotamento profissional; Técnicos de enfermagem; Trabalho noturno.

\section{PREVALENCE OF THE BURNOUT SYNDROME AND SLEEP QUALITY OF NURSING TECHNICAL WORKERS}

ABSTRACT: The Burnout Syndrome and bad sleep quality have been on the increase among nursing technicians. Current analysis, comprising 47 night shift nursing technicians, assesses the prevalence of the Burnout Syndrome and sleep quality. The following questionnaires were applied: (1) socio-economic questionnaire and (2) MBI - Maslach Burnout Inventory and 3 PSQI - Pittsburgh Sleep Quality Index. Results demonstrated that $16,33 \%$ had possible Burnout signs and symptoms; $61,73 \%$ had high trends for the manifestation of Burnout manifestation; $21,93 \%$ had low index for the pathology. In the case of Sleep Quality during the previous month, $74,4 \%$ bad sleeping quality and $25,5 \%$ a good sleeping quality. The occurrence of the Burnout Syndrome and bad sleeping quality in the population under analysis was high, probably due to night shift and working periods up to three shifts for better salaries. Awareness of the importance of sleep and habits that lessen stress may be a solution to mitigate the side effects in one's personal and professional life.

KEY WORDS: Burnout, Professional; Licensed practical nurses; Night work . 


\section{INTRODUÇÃO}

A jornada de trabalho pode se tornar elemento que propicia desgaste e sofrimento ao trabalhador. A busca incessante pela realização de diferentes atividades provoca no homem um aumento das cargas laborais, levando ao aparecimento de doenças físicas, psíquicas e emocionais. (RIBEIRO et al., 2012).

O hospital é uma organização complexa, cujo processo de trabalho depende da conjugação de saberes de diferentes categorias profissionais Nestes locais, o trabalho da enfermagem, integrante do processo de trabalho em saúde, compõe-se das dimensões assistencial, predominantemente desenvolvidas pelos auxiliares e técnicos de enfermagem, sob a supervisão dos enfermeiros (ROCHA et al., 2014).

Neste processo de trabalho, os profissionais de saúde são acometidos por diversos problemas de saúde relacionados ao trabalho em razão da exposição aos riscos e cargas inerentes à profissão (SARQUIS et al., 2013). Atualmente a escala de trabalho mais adotada para as equipes de enfermagem no Brasil são em turnos de 12 horas de trabalho diário (noturno), seguido de 36 horas de descanso.

Com o aumento da carga e jornadas duplas de trabalho a redução do tempo de dormir tornou-se um hábito comum. Geralmente trabalhadores no âmbito hospitalar estão mais expostos a diversos agentes estressores como, por exemplo, jornadas duplas de trabalho, tensão, baixa remuneração e pouco tempo para dormir, exigindo maior controle de suas emoções (COSTA; MARTINS, 2011). O estresse pode ser agudo ou crônico e as consequências de altos níveis de estresse crônico são percebidas, na esfera pessoal, pelo absenteísmo, queda de produtividade, desmotivação, dificuldades interpessoais, doenças físicas variadas, depressão, ansiedade e infelicidade (SADIR; BIGNOTTO; MARILDA, 2010).

No âmbito do trabalho, as consequências do estresse podem incluir, ainda, falta de ânimo, falta de envolvimento com os trabalhadores de enfermagem que trabalham em hospitais. Além da maneira como eles conseguem suportar situações desgastantes, principalmente pela constante convivência com o sofrimento, dor, a morte e tantos outros sentimentos e reações desencadeadas pelo processo de doença (DALRI et al., 2014).

Pesquisas realizadas por Lancman e Sznelwar (2004), levam a defender a idéia de que a qualidade e o estilo de vida estão diretamente relacionados à qualidade do sono. Desta forma, os trabalhadores noturnos são privados de uma qualidade de vida.

Segundo Moustaka e Constantinidis (2010), enfermeiros estressados estão mais susceptíveis à ocorrência de acidentes e enfermidades relacionadas ao trabalho e podem, ainda, desenvolver suas atividades de forma ineficiente, resultando certamente em consequências negativas ao indivíduo e/ou à população assistida. Há outro agravante para estes trabalhadores, as duplas jornadas de trabalho e na maioria das vezes em turno noturno, levando a privação de sono.

Os profissionais que exercem seus trabalhos noturnamente estão mais expostos aos distúrbios e patologias do sono, bem como aumento da sonolência diurna. A privação do sono durante a noite provoca alterações no ciclo vigília-sono, o que interfere no rendimento físico e mental do indivíduo, repercutindo na maior parte dos casos em prejuízos emocionais, físicos, sociais e profissionais (ROCHA; MARTINO, 2010).

O ciclo vigília-sono é um dos ritmos circadianos biológicos mais evidentes do ser humano que difere a alternância do dia-noite, claro-escuro. É organizado temporalmente por estruturas internas que se localizam no sistema nervoso, que lhe conferem em condições naturais, um padrão cíclico básico de 24 horas, no ciclo vigília ocorrem a produção da melatonina que são produzidas na glândula pineal, esta é sintetizada quando a noite chega e o cortisol, produzido na glândula supra renal, é sintetizado quando inicia o dia (MENDES; MARTINO, 2012).

Estresses no trabalho e uma má qualidade de sono levam muitas vezes os trabalhadores a desenvolver a Síndrome de Burnout (SB), que é caracterizada como uma exaustão emocional, despersonalização e baixa realização profissional. A Síndrome de Burnout, ou Síndrome do Esgotamento Profissional, é um distúrbio psíquico descrito em 1974 por Freudenberger, um médico americano. O transtorno está registrado no Grupo V da CID-10 (Classificação Estatística Internacional de Doenças e Problemas Relacionados à Saúde). Suas 
principais características são o estado de tensão emocional e estresse crônico, provocados por condições de trabalho físicas, emocionais e psicológicas desgastantes.

A síndrome se manifesta especialmente em pessoas cuja profissão exige envolvimento interpessoal direto e intenso com outras pessoas. Profissionais das áreas de educação, saúde, assistência social, recursos humanos, agentes penitenciários, bombeiros, policiais e mulheres que enfrentam dupla jornada de trabalho correm risco maior de desenvolver o transtorno, conforme descrevem MUROFUSE; ABRANCHES; NAPOLEÃO, 2005.

O estresse é reconhecido pelo Ministério da saúde como um grave problema de saúde pública, pois estima-se que milhares de pessoas em todo o mundo estejam com a Síndrome de Burnout, reconhecida também como Síndrome do Esgotamento Profissional (MINISTÉRIO DA SAÚDE BRASIL, 2001).

No Brasil, poucas são as pesquisas realizadas sobre o assunto, porém os dados são preocupantes, uma pesquisa realizada pela International Stress Management Association (ISMA-BR, 2006), aponta que 70\% dos brasileiros sofrem as consequências do estresse, desses, $30 \%$ são vítimas do Burnout. Estima-se que em 2007, foram afastados do trabalho 4,2 milhões de indivíduos, sendo que em 3.852 foram diagnosticados Síndrome de Burnout (GONÇALVES, 2008).

A prevenção continuada é a melhor forma de vencer a Síndrome de Burnout. O desenvolvimento de métodos, orientação e treinamento para prevenção de disfunções e distúrbios emocionais deve ser meta centrada para os profissionais da saúde (COSTA, 2007).

Este estudo se faz importante, uma vez que tem como objetivos identificar a prevalência da Síndrome de Burnout e a Qualidade de Sono em técnicos de enfermagem que trabalham no turno noturno.

\section{METODOLOGIA}

Este é um estudo observacional transversal, quantitativo. Realizado com 47 técnicos de enfermagem.

Foi realizado um primeiro contato com os gestores, responsáveis pelo hospital, e as enfermeiras(os) das equipes de enfermagem de diversos setores do turno da noite, a fim de esclarecer o objetivo do trabalho e requerer suas autorizações.

Os participantes foram esclarecidos sobre o estudo. Os critérios para inclusão dos indivíduos na pesquisa foram: concordância em participar da investigação e prontificação em responder aos questionários.

O estudo foi realizado com técnicos de enfermagem do período noturno do hospital Santa Casa do município de Campo Mourão, Paraná.

Foi aplicado a cada participante um questionário sócio econômico, baseado em um conjunto de questões objetivas e de múltipla escolha, com o objetivo de avaliar parâmetros sociais e econômicos, bem como, sexo, estado civil, escolaridade e condições de trabalho.

Em seguida, aplicou-se a cada participante um questionário de avaliação auto-aplicável, para avaliar a Síndrome de Burnout foi utilizado o MBI Maslach Burnout Inventory, de criação de Maslach e Jackson, (1986), que tem a tradução validada para a língua portuguesa, contendo questões que identificam as dimensões sintomatológicas do Burnout. Esse instrumento avalia as três dimensões da síndrome: Exaustão Emocional (3 itens), Realização Pessoal no Trabalho (11 itens) e Despersonalização (3 itens). Totalizam, portanto, 17 itens.

Ainda utilizou-se como instrumento de estudo um questionário de Qualidade de Sono, o Pittsburgh Sleep Quality Index - PSQI, que possibilita avaliar as características dos padrões de sono e quantificar a qualidade do sono do indivíduo, esta escala é composta por perguntas relativas aos seus hábitos de sono durante o último mês, as questões foram abertas e fechadas de múltipla-escolha e discursivas. As pontuações destes componentes são então somadas para produzirem um escore global, que varia de 0 a 21, onde, quanto maior a pontuação, pior a qualidade do sono. Um escore global do PSQI > 5 indica que o indivíduo está apresentando grandes dificuldades, sendo a pontuação máxima de 21 pontos. De 0 a 4 indica boa qualidade de sono, de 5 a 10 refere-se a uma qualidade ruim do sono e acima de 10 pontos trata-se de distúrbios do sono. Os escores superiores a cinco pontos indicaram qualidade ruim no padrão do sono. Os dados foram analisados e comparados às médias dos escores obtidos pelos técnicos de enfermagem com os escores das escalas. 
A análise foi feita atribuindo escores para cada questão de acordo com as instruções já determinadas pelos questionários.

Para a Síndrome de Burnout, as respostas são avaliadas por meio de uma escala de frequência de 5 pontos, variando de 0 (nunca) a 5 (frequentemente). $\mathrm{O}$ MBI é o instrumento mais utilizado para mensuração da Síndrome Burnout.

A definição operacional padrão da Síndrome de Burnout ao avaliar fatores como exaustão emocional, despersonalização e ineficácia. A exaustão representa o componente individual, com sentimentos de ser exigido para além de seus recursos. A despersonalização se refere ao componente interpessoal e, em níveis altos, pode dar uma impressão inicial de defesa e proteção, mas com risco de cronificação do distanciamento. A ineficácia é o componente de autoavaliação, normalmente acompanhada de sentimentos de incompetência e baixa produtividade (TIRONI et al., 2016).

Para tabulação, processamento e análise, os dados obtidos relativos aos instrumentos MBI e PSQI foram submetidos a análises descritivas da frequência relativa (quantidades de entrevistados) e frequência absoluta (porcentagem dos resultados) conforme tabelas 1 e 2, utilizando os programas Word, e EpInfo versão 3.3.2.
Os critérios foram esclarecidos aos sujeitos, quando se apresentou o Termo de Consentimento Livre e Esclarecido (TCLE). Antes de assiná-lo, os participantes tomaram ciência da confidencialidade, do anonimato e do direito de desistência em qualquer etapa do estudo, sem que houvesse prejuízo, dano ou constrangimento durante sua participação.

O estudo realizou-se no período de dezembro de 2015 a fevereiro de 2016, aprovado pelo Comitê Permanente de Ética em Pesquisa (COPEP) da Universidade Estadual de Maringá (UEM) (41853).

\section{RESULTADOS}

Analisando os dados sócio-demográficos observou-se a predominância do sexo gênero feminino 91,1\% para 8,1\% masculino. A faixa etária média de idade foi de 35 anos. Da amostra, 70,2\% estão em união estável, $25,5 \%$ solteiros. E ainda, $89 \%$ exercem dupla jornada de trabalho.

Com relação à escolaridade $78,7 \%$ possuem ensino médio completo. Quanto à atividade profissional, todos são formados em técnicos de enfermagem, sendo que a maioria $(55,4 \%)$ desempenha outra função, além de sua qualificação. A média de tempo de trabalho na profissão é 17 anos (Tabela 1).

Tabela 1. Variáveis sociodemográficas de técnicos de enfermagem do período noturno do Hospital Santa Casa de Campo Mourão, Paraná (2016)

\begin{tabular}{lcc}
\hline Variáveis & Frequência Relativa F.R. & Frequência Absoluta F.A. \\
\hline Sexo & & \\
Feminino & 43 & 91,1 \\
Masculino & 04 & 8,1 \\
\hline Total & 47 & 100,0 \\
\hline Estado civil & 12 & 25,5 \\
Solteira & 33 & 70,2 \\
Casada & 02 & 4,3 \\
Outro & 47 & 100,0 \\
\hline Total & & \\
\hline Escolaridade & 37 & 78,7 \\
Ensino médio completo & 2 & 4,3 \\
Ensino médio incompleto & 1 & 2,1 \\
Ensino superior completo & 7 & 14,9 \\
Ensino superior incompleto & 47 & 100,0 \\
\hline Total & & \\
\hline
\end{tabular}


Dos entrevistados, 16,33\% apresentaram possíveis sinais e sintomas de Burnout; $61,73 \%$ possuíam alto índice para manifestação de Burnout; e 21,93\% apresentaram baixo índice para apresentação da patologia. Verificou-se que $70,2 \%$ dos profissionais apresentaram média classificação para exaustão emocional; 57,5\% média classificação para despersonalização; e 57,5\% apresentaram média classificação para realização profissional, características que estabelecem possíveis manifestações de Burnout (Figura 1).

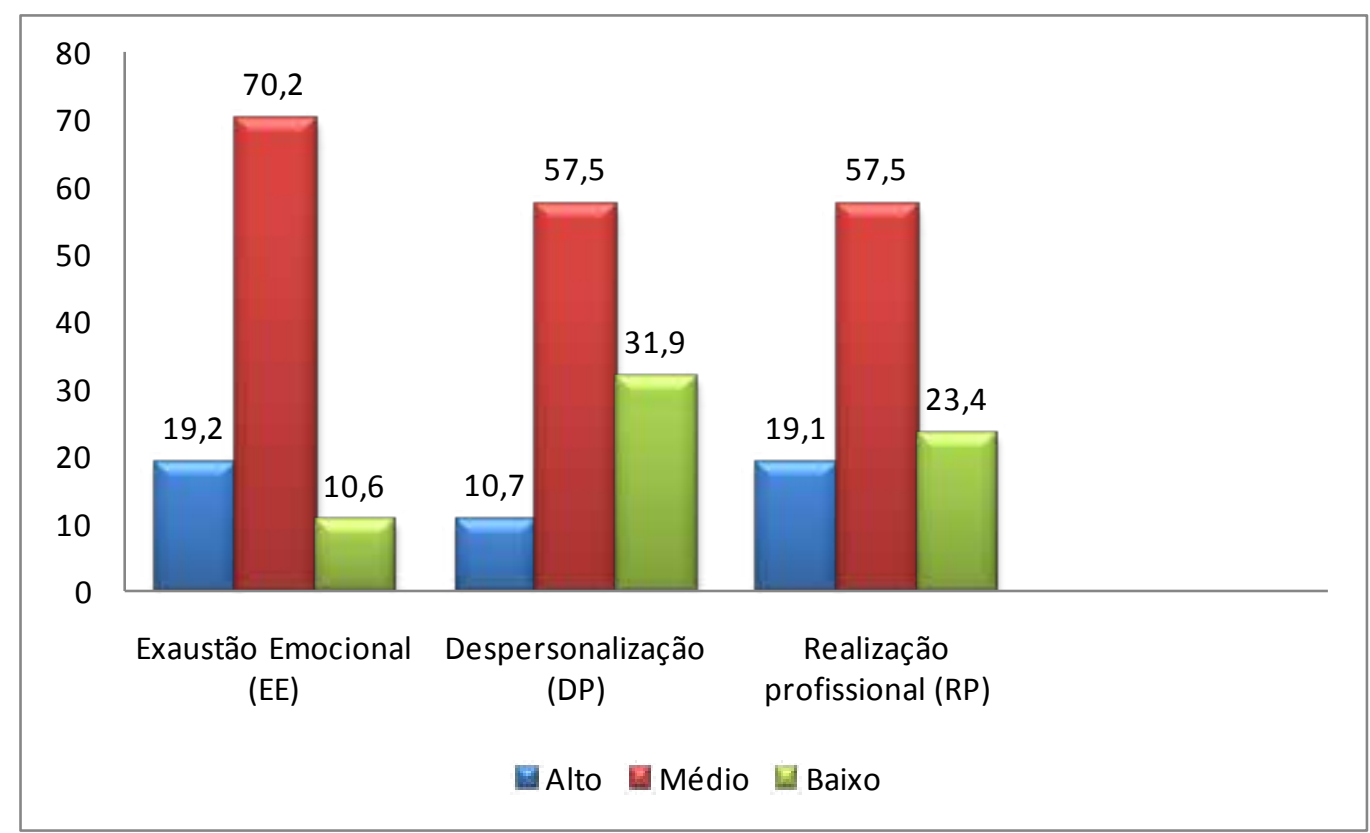

Figura 1. Distribuição em \% da manifestações de Sindorme de Burnout, MBI na amostra dos técnicos de enfermagem do turno da noite do Hospital Santa Casa de Campo Mourão, Paraná (2016).

A maioria, 53,2\%, diz nunca ter faltado ao trabalho por problemas de saúde física ou mental, enquanto $23,4 \%$ já necessitaram se afastar do trabalho por problemas de saúde.

Dos pesquisados, 70,2\%, afirmam enfrentar problemas de saúde. Os sintomas mais relatados são: ansiedade, dores em membros, estresse, pânico, dificuldade de concentrar-se, hipertensão, diabetes, problemas renais, labirintite e problemas cardíacos.

Observamos que, $59,5 \%$ tiveram cansaço ou esgotamento frequente nos últimos seis meses, sendo que $48,9 \%$ apresentaram dificuldades para dormir.

Ao analisar a qualidade do sono, verificouse que, dos técnicos de enfermagem participantes da pesquisa, 25,6\% possuem boa qualidade do sono e 74,5\% apresentam má qualidade de sono conforme o Pittsburgh Sleep Quality Index - PSQI, que possibilita avaliar as características dos padrões de sono (tabela 2).
Tabela 2. Distribuição em \% da frequencia de escores obtidos por meio da aplicação do Índice de Qualidade do Sono (PSQI), em técnicos de enfermagem do Hospital Santa Casa de Campo mourão Paraná (2016)

\begin{tabular}{lcc}
\hline Pontuação do PSQI & $\begin{array}{c}\text { Frequência } \\
\text { Relativa } \\
\text { F.R. }\end{array}$ & $\begin{array}{c}\text { Frequência } \\
\text { Absoluta } \\
\text { F.A. }\end{array}$ \\
\hline PSQI $>$ 5 & 35 & 74,5 \\
PSQI $<5$ & 12 & 25,5 \\
\hline Total & 47 & 100,0 \\
\hline
\end{tabular}

Dos técnicos de enfermagem entrevistados, $78,8 \%$ dormem em média de 6 a 8 horas por noite.

Quando perguntado sobre a qualidade de sono no último mês, 74,5\% classificaram seu sono como bom e $25,5 \%$ o classificaram como ruim. A maioria dos pesquisados, 59,6\%, não fazem uso de medicação para dormir.

Os técnicos de enfermagem responderam que no último mês, seu entusiasmo e ânimo para realizar 
atividades habituais foram leves para 36,2\%; razoáveis para 29,8\% dos participantes da pesquisa; e um grande problema para $8,5 \%$ dos entrevistados.

\section{DISCUSSÃO}

O presente estudo foi predominado pelo sexo feminino, o que nos apresenta a preferência do sexo feminino para a profissão de técnico de enfermagem. Em nossa amostra, o índice de profissionais que exercem dupla jornada de trabalho é bastante elevado.

À situação de trabalho, os vários turnos assumidos pelos profissionais possivelmente decorrem de suas condições pessoais, laborativas e financeiras, levando-os a depender de mais de um emprego e ficando expostos a diversos fatores de riscos desencadeadores de estresse (LIMA et al., 2013). O que pode gerar danos à qualidade de vida e ao bom desempenho profissional.

Para Dias (2014), a síndrome de Burnout assume atualmente uma importância crescente, atraindo a atenção da comunidade científica pelo impacto que causa nos trabalhadores de diferentes profissões, em especial, nos enfermeiros e nos médicos, devido à severidade das suas consequências, assumindo-se como uma problemática psicossocial relevante para a saúde do trabalhador.

Ao analisar a faixa etária, observa-se a predominância de jovens com maiores níveis de Burnout e má qualidade do sono. O que difere de nossos achados, onde a idade média foi de 35 anos. Para os pesquisadores, este fato se da muitas vezes pelas frequentes frustrações profissionais, pois muitas vezes pode tratar-se de recém formados que não possuem muita confiança e qualificação profissional que a área exige, tornando o indivíduo instável em seu trabalho, acarretando medo, contribuindo assim com o agente estressor, para Síndrome de Burnout e a má qualidade do sono (CARLOTTO; CÂMARA, 2008).

Com relação ao fenômeno psicossocial, a Síndrome de Burnout ocorre como resposta ao estresse laboral crônico e reflete importante desafio da vida profissional no século XXI. Profissionais que lidam com situações de emergência, por entrarem em contato com eventos traumáticos, estão mais suscetíveis ao comprometimento de seu bem-estar físico e emocional (MELO; CARLOTTO, 2016).
Conforme, Moreira et al., (2012) observam ainda que nas pesquisas relacionadas ao tema encontrase dificuldades para comparar resultados de estudos brasileiros, visto que a maioria dos estudos realizados sobre Burnout são norte-americanos. Esta posição, mostra que são poucas publicações encontradas para a realização deste ensaio, nos mostrando a importância de correlacionar a em relação à Síndrome de Burnout e a Qualidade do Sono, principalmente nos ambientes hospitalares

Ao analisar as três dimensões da Síndrome de Burnout, foram coletados os dados referentes ao (MBI), observando a importância que o desgaste emocional que envolve o sentimento de esgotamento físico e mental. $\mathrm{Na}$ nossa pesquisa, apenas 10,6\% apresentaram uma baixa exaustão emocional, isto é, a maioria está extremamente desgastada emocionalmente e fisicamente, o que certamente, causa conflitos no trabalho em casa e uma interferência direta na qualidade do sono.

Fazendo um paralelo com a realização profissional, 23,4\% dizem estar com baixa satisfação, isto nos mostra uma relação muito próxima com sentir-se bem emocionalmente e trabalhar realizado. Ainda há a despersonalização, que são as atitudes negativas, para com as pessoas, principalmente com os pacientes dos serviços prestados pelos profissionais. Na nossa análise, apenas $10,7 \%$ demonstraram não terem essas atitudes, o que nos leva a constatar os escores elevados nas dimensões da síndrome, evidenciando a alta probabilidade dos pesquisados em desenvolver a Síndrome de Burnout.

O burnout é uma síndrome psicológica, de esgotamento profissional, decorrente da sobrecarga emocional crônica no trabalho que envolve relacionamento interpessoal de grande responsabilidade e apresenta três dimensões interdependentes: exaustão emocional, despersonalização e ineficácia (TRIGO, 2010). Importante esclarecer que a Síndrome de Burnout não é um problema do indivíduo, mas do ambiente social no qual desempenha suas atividades.

Murofuse, Abranches e Napoleão, (2011), salientam que a Síndrome de Burnout é um grande problema psicossocial atual, despertando o interesse e a preocupação das entidades governamentais, empresariais e sindicais norte-americanas e européias, 
devido à severidade de suas consequências, tanto em nível individual como organizacional. O sofrimento do indivíduo traz consequências tanto para sua saúde quanto para seu desempenho profissional, pois passam a existir alterações e ou disfunções pessoais e organizacionais, com repercussões econômicas e sociais. Ainda, para estes autores, a enfermagem, como prática social, não ficou isenta às transformações introduzidas no mundo do trabalho.

Barboza et al., (2008), observaram que 88\% dos profissionais auxiliares de enfermagem possuem má qualidade de sono. Em estudo realizado por Souza, (2007), verificou-se que os turnos da noite são grandes indícios para o desenvolvimento de distúrbios do sono e má qualidade do mesmo. Devido ao pouco tempo de sono, muitas vezes os trabalhadores não dormem regularmente o tempo necessário para repor suas energias.

O trabalho noturno, por estar associado a um cotidiano essencialmente diferente daquele adotado pela sociedade em geral, acarreta muitas vezes problemas de saúde que aparecem muito mais nos trabalhadores noturnos do que nos diurnos como problemas cardíacos, renais, diabetes hipertensão e muitas dores. Podendo, ainda, acarretar muitas outras consequências à saúde dos trabalhadores (ROTENBERG et al., 2001).

O Índice de Qualidade do Sono varia de acordo com o tempo médio de sono, pois profissionais que trabalham no turno da noite tem uma predisposição maior para conseguir relaxar e ter uma soma que reflita em seu sono, chegando a um número mínimo de oito horas de sono que é preconizado por estudos, como o tempo que seria ideal para repor as energias e deixar de enfrentar certas patologias. Pessoas com histórico de vida profissional com pouco tempo para dormir e repor as energias apresentam uma qualidade de sono ruim em comparação trabalhadores diurnos (MARQUEZE; JUST; MORENO, 2009).

Recomenda-se que a equipe de enfermagem do turno noturno possa ter oportunidades de tirar um tempo para descanso e cochilos, o que contribuiria para o auxílio na recuperação de energias dissipadas ou compensar o déficit de sono durante suas atividades noturnas. O desenvolvimento, a promoção e implantação de políticas de saúde nos locais de trabalho é uma questão de responsabilidade à saúde do trabalhador.

\section{CONCLUSÃO}

É de suma importância identificar a ocorrência de Síndrome de Burnout e observar a Qualidade do Sono em técnicos de enfermagem para que não ocorra a diminuição da qualidade do trabalho bem como a qualidade de vida pessoal de cada indivíduo.

No hospital estudado os índices de Síndrome de Burnout foram de 74,5\% e os índices de má Qualidade do Sono de $61,73 \%$. Ambos índices são considerados alto para a amostra estudada.

Os dados obtidos no estudo poderão ser úteis para a Direção do Hospital Santa Casa de Campo Mourão, contribuindo para melhoria da qualidade de vida e segurança no trabalho da equipe de técnicos de enfermagem, bem como dos serviços prestados por este órgão.

A partir destes resultados, sugere-se acompanhamento anual desses trabalhadores, através de intervenções para que não surjam patologias à saúde desses empregados proporcionados pela síndrome nesse estudo pesquisado, bem como o aprimoramento de educação continuada destes profissionais, por meio de palestras educativas, baseadas nas análises de risco e potencial de aparecimento dessas problemáticas.

Sugere-se, no entanto, continuidade em pesquisas nessa área da influência do trabalho em turnos da noite na saúde ocupacional dos trabalhadores, uma vez que a influência do turno de trabalho e cronotipo na qualidade de vida dos trabalhadores de enfermagem perpassam e influenciam na sua qualidade de trabalho.

\section{REFERÊNCIAS}

BARBOZA, J. I. R. A.; MORAES, E. L.; PEREIRA, E. A.; REIMÃO, R. N. A. A. Avaliação do padrão de sono dos profissionais de Enfermagem dos plantões noturnos em Unidades de Terapia Intensiva. Einstein, São Paulo, v. 3, p. 296-301, 2008.

BRASIL. Ministério da Saúde. Doenças relacionadas ao trabalho: manual de procedimentos para o serviços de saúde. Brasília: Ministério da Saúde; OPAS/OMS, 2001. 
CARLOTTO, M. S.; CÂMARA, S. G. Análise da produção científica sobre a Síndrome de Burnout no Brasil. PSICO, Porto Alegre, PUCRS, v. 39, n. 2, p. 152-158, 2008.

CARLOTTO, M. S.; PALAZZO, L. S. Síndrome de Burnout e fatores associados: um estudo epidemiológico com professores. Cad Saúd Públ, v. 22, n. 5, p. 1017-1026, 2006.

COSTA, A.L.S. Stress in nursing students: construction of determining factors. REME, v. 11, p. 414-19, 2007.

COSTA, D.T.; MARTINS, M.C.F. Estresse em profissionais de enfermagem: impacto do conflito no grupo e do poder do médico. Revista esc. enfermagem, USP. v. 45, n. 5, p. 1191-1198, 2011.

DALRI, R.C.M. B.; SILVA, L.A.; MENDES, A. M. O.; ROBAZZI, M.L.C. Carga horária de trabalho dos enfermeiros e sua relação com as reações fisiológicas do estresse. Rev Lat Am Enfermagem, v. 22, n. 6, p. 959-965, 2014.

DIAS, S. Síndrome de burnout: um estudo comparativo entre enfermeiros e médicos portugueses. Diaphora, $v$. 12, n. 2, p. 35-41, 2014.

GONÇALVES, E. Síndrome de Burnout: desconhecida, mas perigosa. Folha de Londrina, mar .17. Caderno 2, p. 7.2008.

INTERNATIONAL STRESS MANAGEMENT ASSOCIATION - ISMA-BR. Trabalho, stress e saúde: prevenindo o Burnout: da teoria à ação. 2006. Disponível em: $\quad<$ http://www.ismabrasil.com.br/tpls/147. asp?idPagina $=49 \& \mathrm{idPg}=601 \& \mathrm{mAb}=\mathrm{n}>$. Acesso em: 02 set. 2015.

LANCMAN. S.; SZNELWAR, L.I. (Org.). Christophe Dejours: da psicologia à psicodinâmica do trabalho. Brasilia: Fiocruz, 2004.

LIMA, M.B.; SILVA, L.M.S.; ALMEIDA, F.C.M.; TORRES, R.A.A; DOURADO, H.H.M. Stressors in nursing with double or more working hours. R. pesq.: cuid. fundam, v.5, n.1, p.3259-3266, 2013.

MARQUEZE, E.C.; JUST, M.S.; MORENO, C.R.C. Qualidade de sono, atividade física durante o tempo de lazer e esforço físico no trabalho entre trabalhadores noturnos de uma indústria cerâmica. Revista brasileira de sáude ocupacional, v. 34, n. 119, p. 93-100, 2009.

MELO, L.P.; CARLOTTO, M.S. Prevalência e preditores de Burnout em bombeiros. Psicol. cienc. prof., v.36, n.3, 2016.

MENDES, S.S.; MARTINO, M.M.F. Trabalho em turnos: estado geral de saúde relacionado ao sono em trabalhadores de enfermagem. Revista esc. enfermagem USP, v. 46, n. 6, p. 1471-1476, 2012.

MOREIRA, D.S.; MAGNAGO, R.F.; SAKAE, T.M.; MAGAJEWSKI, F.R.L. Prevalência da síndrome de burnout em trabalhadores de enfermagem de um hospital de grande porte da Região Sul do Brasil. Cad Saude Publica, v. 25, n. 7, p.1559-1568, 2012.

MOUSTAKA, E.; CONSTANTINIDIS, T.C. Sources and effects of Work-related stress in nursing. Health Sci J., v. 4, n. 4, p. 210-6, 2010.

MUROFUSE, N. T.; ABRANCHES, S. S.; NAPOLEÃO, A. A. Reflexões sobre estresse e Burnout e a relação com a enfermagem. Rev Lat Am Enfermagem, Ribeirão Preto, v. 13, p. 255-261, 2005.

MUROFUSE, N.T.; ABRANCHES, S.S.; NAPOLEÃO, A.A. Reflexões sobre estresse e Burnout e a relação com a enfermagem. Rev Lat Am Enfermagem, Ribeirão Preto, v. 13, n. 2,2011

RIBEIRO, R.P.; MARTINS, J.T.; MARZIALE, M.H.P.; ROBAZZI, M.L.C.C. O adoecer pelo trabalho na enfermagem: uma revisão integrativa. Rev Esc Enferm USP, v.46, n.2, p.495-504, 2012.

ROCHA, F.L.R.; MARZIALE, M.H.P.; CARVALHO, M.C.; ID, S.F.C.; CAMPOS, M.C.T. A cultura organizacional de um hospital público brasileiro. Rev Esc Enferm USP, v. 48, n. 2, p. 308-314, 2014.

ROCHA, M.C.P.; MARTINO, M.M.F. O estresse e qualidade de sono do enfermeiro nos diferentes turnos hospitalares. Rev Esc Enferm USP, v. 44, n. 2, p. 280-286, 2010.

ROTENBERG, L.; PORTELA, L. F.; MARCONDES, W. B.; MORENO, C.; NASCIMENTO, C. P. Gênero e trabalho 
noturno: sono, cotidiano e vivências de quem troca a noite pelo dia. Cad Saude Publica, v. 17 p. 639-649, 2001.

SADIR, M.; BIGNOTTO, M.; MARILDA E. N. Stress e qualidade de vida: influência de algumas variáveis pessoais. Paidéia (Ribeirão Preto), v. 20, n. 45, p. 73-81, 2010.

SARQUIS, L.M.M.; BAPTISTA, P.C.P.; MINIMEL, V.A.; SILVA, F.J.; FELLI, V.E.A. Exposição ao material biológico: consequências para os profissionais de enfermagem. Ciênc. cuid. Saúde, v.12, n.4, out./dez. 2013.

SIEGEL, K.; KNUTSON, K.; LEPROULT, R.; TASALI, E. Sleep loss: a novel risk factor for insulin resistance and type 2 diabetes. J Appl Physiol., v. 99, n. 5, p. 2008-19, 2005.

SOUZA, J. C. Sonolência excessiva em trabalhadores da área de enfermagem. J Bras Psiquiatria. v. 56, n. 3, p. 180-3, 2007.

TIRONI, M. O. S.; TELES, J. M. M.; BARROS, D. S.; VIEIRA, D. F. V. B.; SILVA- FILHO, C. M.; MARTINS-JÚNIOR, D. F.; MATOS, M. A.; NASCIMENTO-SOBRINHO, C. L. Prevalência de síndrome de burnout em médicos intensivistas de cinco capitais brasileiras. Rev Bras Ter Intensiva, v.28, n.3, p.270-277, 2016.

TRIGO, T.R. Síndrome de Burnout ou esgotamento profissional: como identificar e avaliar. In: Glina DM, Rocha LE, organizadoras. Saúde mental no trabalho: da teoria à prática. São Paulo: Roca, p.160-75, 2010.

Recebido em:06 de julbo de 2016 Aceito em: 29 de novembro de 2016 